\title{
First-Principles Study of the Switching Mechanism of [2]Catenane Molecular Electronic Devices
}

\author{
Yong-Hoon Kim, ${ }^{*}$ Seung Soon Jang, Yun Hee Jang, and William A. Goddard III ${ }^{\dagger}$ \\ Materials and Process Simulation Center, California Institute of Technology, Pasadena, California 91125-7400, USA
}

(Received 30 June 2004; published 21 April 2005)

\begin{abstract}
We present a first-principles study of the coherent charge transport properties of bistable [2]catenane molecular monolayers sandwiched between $\mathrm{Au}(111)$ electrodes. We find that conduction channels around the Fermi level are dominated by the two highest occupied molecular orbital levels from tetrathiafulvalene (TTF) and dioxynaphthalene (DNP) and the two lowest unoccupied molecular orbital levels from tetracationic cyclophane (CBPQT ${ }^{4+}$ ), and the OFF to ON switching results from the energetic shifts of these orbitals as $\mathrm{CBPQT}^{4+}$ moves from TTF to DNP. We show that the superposition principle can be adopted for predicting the function of the composite device.
\end{abstract}

PACS numbers: 85.65.th, 68.47.Pe, 73.63.-b, 81.16.Fg

An outstanding recent achievement in molecular electronics is the demonstration of solid-state molecular switch tunnel junctions based on a Langmuir-Blodgett (LB) monolayer of bistable [2] catenane and [2] rotaxane molecules $[1,2]$. The switch is opened and closed by applying a large voltage $(\sim \pm 2 \mathrm{~V})$, and the switching status is read via a small voltage $(-0.2-+0.2 \mathrm{~V})$. It has been demonstrated to be recyclable under ambient conditions, and has been successfully employed for logic and memory circuits within the two-dimensional (2D) crossbar architecture [2]. The switching has been suggested to arise due to the reversible mechanical movement of the tetracationic cyclobis-(paraquat- $p$-phenylene) $\left(\mathrm{CBPQT}^{4+}\right)$ ring between the tetrathiafulvalene (TTF) and the 1,5dioxynaphthalene (DNP) moieties with the former or latter configuration as the switch OFF/ON state. However, while spectroscopy and electrochemistry experiments clearly showed the evidence of $\mathrm{CBPQT}^{4+}$ movement [3], there is no direct confirmation of this interpretation from tunneljunction experiments $[2,4]$.

Herein, we carry out quantum mechanical densityfunctional and matrix Green's function calculations coupled with classical force-field molecular dynamics, and provide a first-principles understanding of the switching mechanism of the [2]catenane device. In a previous paper [5], considering free simplified [2] rotaxane molecules, we identified possible conduction paths and listed plausible switching mechanisms. In another work [6], using an idealized extended [2] rotaxane molecule attached to three $\mathrm{Au}$ atoms as each electrode, we obtained the ON/OFF switching order in agreement with the experimental proposition. In this Letter, we prepare a realistic [2]catenane monolayer configuration, sandwich it between semiinfinite $\mathrm{Au}(111)$ electrodes to prepare a device model, and compute its coherent charge tunneling properties, all within the $2 \mathrm{D}$ periodic boundary condition. Based on the accurate location of molecular levels with respect to the electrode Fermi level, we provide the physical origin of the switching function. We also demonstrate that the "super- position" principle approximately holds in predicting the characteristics of the composite device, thus showing the possibility of the bottom-up building approach in designing complex molecular electronic devices.

The proposed two most important features of the solidstate [2]catenane device are that (i) its two bistable conformations, $\mathrm{CBPQT}^{4+}$ encircling TTF (CBPQT@DNP) and $\mathrm{CBPQT}^{4+}$ encircling DNP (CBPQT@DNP) [Fig. 1(a)], have very different conductance behaviors at a small "read" bias, and (ii) it can be switched between the two states by applying a large "write" bias. The activation of circumrotational backbone movement (ii), which presumably involves charged states [1], should be affected by the details of the molecule, junction, and electrode configuration [4]. The focus of this Letter is item (i), so we will place CBPQT ${ }^{4+}$ around either TTF or DNP and consider their charge transport characteristics in the neutral state. We model each electrode by three-layer $\mathrm{Au}(111)$ atoms and replace the dimyristoylphosphatidic $\left(\mathrm{DMPA}^{-}\right)$anions by $\mathrm{PF}_{6}{ }^{-}$anions.

Careful characterization of reproducible [2]catenane LB films was the important first step of the experimental device construction [7]. Thus, we started by theoretically searching for the optimal [2]catenane monolayer configuration employing the Dreiding force field [8] coupled with charges from the charge-equilibration model [9]. We mimicked the Langmuir film formation process by using a $\mathrm{Au}(111)$ slab as the template: we prepared $3 \times 4,4 \times 4$, $4 \times 5,5 \times 5,5 \times 6$, and $6 \times 6$ supercells of the primitive $\mathrm{Au}(111)$ unit cells, each with three layers of $\mathrm{Au}$ atoms, as the substrate and placed one CBPQT@TTF [2]catenane molecule. Fixing the Au atoms but allowing the full flexibility of the molecule, we performed 50 ps of molecular dynamics simulations ( $1 \mathrm{fs}$ time step) at $300 \mathrm{~K}$ using the Nose-Hoover thermostat [10]. Details of our force-field optimizations and molecular dynamics simulations methods are described elsewhere [11].

The molecular dynamics results show that the optimal packing density is between one molecule per $4 \times 4$ 


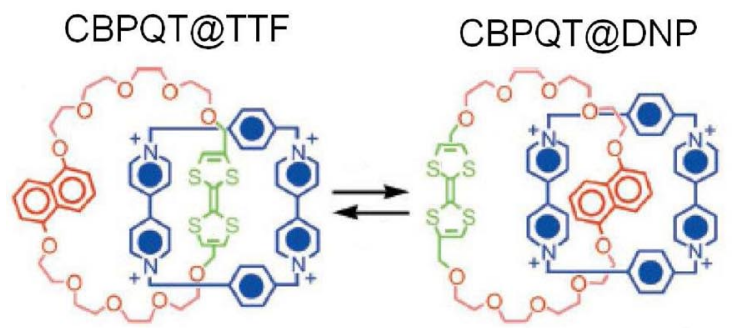

(a)

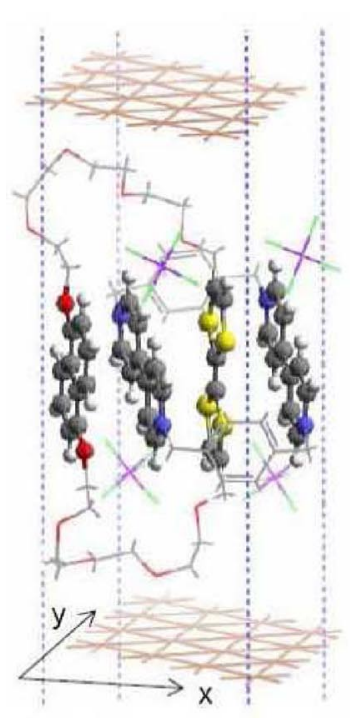

(b)

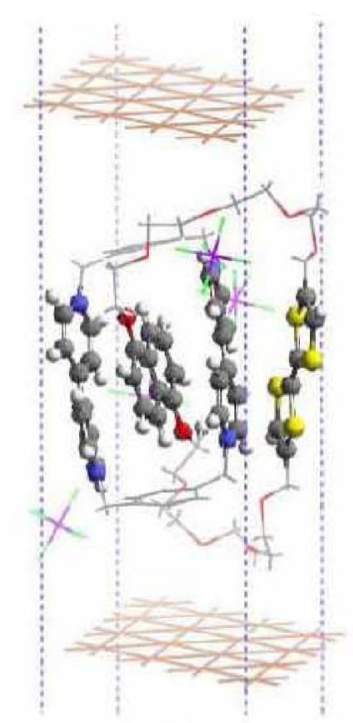

(c)
FIG. 1 (color online). (a) Molecular drawing of the bistable [2]catenane. Geometry of the $4 \times 4-\mathrm{Au}(111)$-cell (b) CBPQT@TTF and (c) CBPQT@DNP [2]catenane monolayers. In (b) and (c), TTF, DNP, two bipyridylium of $\mathrm{CBPQT}^{4+}$, are represented in the ball-and-stick format, and the remainder in the stick format.

$\mathrm{Au}(111)$ cell $\left(115 \AA^{2}\right.$ area) and one per $4 \times 5$ cell $\left(144 \AA^{2}\right.$ area), in good agreement with the experimental value of $120 \AA^{2}$ Langmuir limiting area [7]. In order to complete the device model, we capped the $4 \times 4-\mathrm{Au}(111)$-cell monolayer with another three-layer $\mathrm{Au}(111)$ electrode. The electrode-electrode gap size of $22 \AA$ was chosen such that the center of mass of the molecule is located approximately at the middle of the gap. The molecular conformation of a $4 \times 4-\mathrm{Au}(111)$-cell CBPQT@TTF [2]catenane monolayer is shown in Fig. 1(b). The same procedure was used to obtain a $4 \times 4-\mathrm{Au}(111)$-cell CBPQT@DNP monolayer [Fig. 1(c)]. Atomic coordinates of the two models can be found at the EPAPS depository [12].

For the [2]catenane monolayer device models prepared above, we performed 2D density-functional calculations [13] within the Perdew-Burke-Enzhrof flavor of generalized gradient approximation (GGA) [14]. We adopted norm-conserving pseudopotentials $[15,16]$ and a Gaussian basis set of double- $\zeta$-plus-polarization quality for the [2]catenane and single- $\zeta$-plus-polarization quality for the Au optimized for the GGA pseudopotentials. A single reciprocal-space $\Gamma \vec{k}$ point was sampled.

With the Hamiltonian matrix from the self-consistent density-functional calculation, we next carried out the matrix Green's function calculation to compute the transmission function $[17,18]$,

$$
\begin{aligned}
& T(E)=\operatorname{Tr}\left[\Gamma_{1}(E) G_{M}(E) \Gamma_{2}(E) G_{M}^{+}(E)\right] ; \\
& G_{M}(E)=\left(E S_{M}-H_{M}+\Sigma_{1}+\Sigma_{2}\right)^{-1} ; \\
& \Sigma_{a}=x_{a} g_{S a} x_{a}^{+} ; \quad \Gamma_{a}=i\left(\Sigma_{a-} \Sigma_{a}^{+}\right) \quad(a=1,2),
\end{aligned}
$$

where $S_{M}$ and $H_{M}$ are the molecule parts of the overlap and Hamiltonian matrices, and $x_{a}$ are the molecule-electrode $a$ parts of the total $E S-H$ operator. The self-energies $\Sigma$ provide the $a b$ initio values of the broadening and shift of the molecular energy levels due to the coupling of molecules to electrodes. The surface Green's functions $g_{S}$ were extracted from two independent bulk GGA calculations with the unit cells corresponding to the top and bottom electrodes with the single- $\vec{k}_{\|}$-point sampling along the electrode-surface direction and the two- $\vec{k}_{\perp}$-point sampling along the electrode-normal direction. The energy was first scanned around the Fermi level of the system $E_{\mathrm{F}}$ using $0.02 \mathrm{eV}$ steps, and then the resolution was increased near the transmission resonances up to $10^{-4} \mathrm{eV}$.

The main objective of the current study is understanding the charge conduction behavior of [2]catenane molecules at a small probe voltage or the "remnant molecular signature" that was used to interrogate the switching status $[1,2]$. In the experiments, [2]catenane molecules were separated from the poly-Si bottom electrode and the $\mathrm{Ti} / \mathrm{Al}$ top electrode by, respectively, a $\mathrm{SiO}_{2}$ layer and long DMPA ${ }^{-}$molecules [1]. This implies that the actual electrostatic potential drop over the [2]catenane molecules will be even smaller than the probe voltage. Thus, the zerobias transmissions should provide sufficient information on the switching status.

Transmission functions of the $4 \times 4-\mathrm{Au}(111)$-cell device in the CBPQT@TTF and CBPQT@DNP configurations are shown in Fig. 2(a). The prominent feature common to both is four sharp resonances near (two below and two above) $E_{\mathrm{F}}$, which indicates that the frontier-orbital characters of molecules are well preserved in the device setting and the measurement of remnant molecular signature should be meaningful. Note that, since we incorporate a large number of $\mathrm{Au}$ atoms to model the electrode, the relative location of molecular levels with respect to $E_{\mathrm{F}}$ is accurately estimated. Comparing the CBPQT@TTF and CBPQT@DNP cases, we observe that the highest occupied molecular orbital (HOMO) peak lies much lower in CBPQT@TTF than in CBPQT@DNP. 
After obtaining the transmission function, we calculated the current-voltage $(I-V)$ characteristics via the LandauerButtiker formula $[17,18]$,

$$
I(V)=\frac{2 e}{h} \int_{\mu_{1}}^{\mu_{2}} d E T(E, V)\left[f\left(E-\mu_{1}\right)-f\left(E-\mu_{2}\right)\right]
$$

where $\mu_{1}$ and $\mu_{2}$ are the chemical potentials of the bottom and top electrodes, and $f$ is the Fermi-Dirac distribution function. The $I-V$ data obtained within the approximation of $\mu_{1}=E_{\mathrm{F}}$ and $\mu_{2}=E_{\mathrm{F}}+e V$ are shown in Fig. 2(b). The difference of the HOMO transmission characteristics results in the assignment of CBPQT@DNP/TTF as the switch ON/OFF state in agreement with the original experimental proposition [1]. The switching ratio at the bias voltage $-0.2 \mathrm{~V}$ is about $10^{4}$. Conformational fluctuations will likely reduce this ratio to the experimental value of $2-10$. The observed reversal of the switching status below $-0.35 \mathrm{~V}$ suggests that the switching characteristics might be masked at a large bias [19]. We mention that the same assignment of the switching status is also obtained when we probe the lowest unoccupied molecular orbital (LUMO) transmissions. However, because the energetic location of unoccupied orbitals is expected to have been downshifted and thus unreliable within the GGA due to its self-interaction error and the related band gap underestimation [20], here we do not assign the unoccupied orbitals as the levels determining the switching characteristics.

The four dominant conductance channels near $E_{\mathrm{F}}$ of Fig. 2(a) were characterized by calculating the projected density of states (PDOS) of TTF, DNP, and CBPQT ${ }^{4+}$ [Figs. 2(c) and 2(d)]. We find in both CBPQT@TTF and CBPQT@DNP configurations that the first HOMO, the second HOMO, and the two LUMOs are mainly associated with TTF, DNP, and CBPQT ${ }^{4+}$, respectively. The origin of transmission peaks are further analyzed via segment models below.

Taking several other models with different monolayer packing densities and electrode-electrode gap distances, we consistently found that the HOMO transmission peak position in CBPQT@TTF is lower than that in CBPQT@DNP by $0.1-0.3 \mathrm{eV}$. To further confirm its universal nature, we also considered a device model based on the $\mathrm{Cu}(111)$ electrodes with $141 \AA^{2}$ unit cell area and $22 \AA$ gap size. Independent of the upward shift of $E_{\mathrm{F}}$ by $\sim 0.4 \mathrm{eV}$ compared with the Au-electrode case, we found that the relative shift of the HOMO transmission peak between CBPQT@TTF and CBPQT@DNP shows a qualitatively identical behavior.

One interesting question we raised in Ref. [5] is the possibility of designing complex molecular electronic devices in the bottom-up assembly approach. To address this question and obtain a more detailed understanding of the switching mechanism as well as the monolayer packing effect, we now consider simplified segment models based on (I) TTF backbone, (II) TTF backbone encircled by
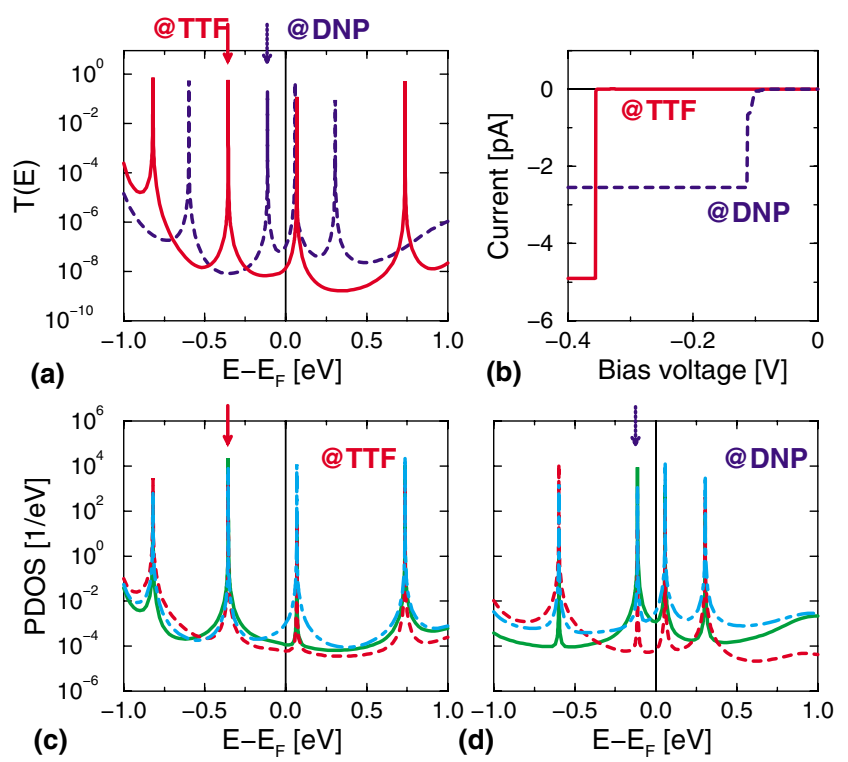

FIG. 2 (color online). (a) Transmissions of the CBPQT@TTF (solid lines) and CBPQT@DNP (dashed lines) [2]catenane monolayers, and (b) their $I-V$ characteristics. Corresponding TTF (solid lines), DNP (dashed lines), and $\mathrm{CBPQT}^{4+}$ (dotdashed lines) PDOS within the (c) CBPQT@TTF and (d) CBPQT@DNP configurations. For clarity, the monotonous $\left(\mathrm{PF}_{6}{ }^{-}\right)_{4} \mathrm{PDOS}$ is not shown.

$\left(\mathrm{CBPQT}^{4+}\right)\left(\mathrm{PF}_{6}^{-}\right)_{4}$, (III) DNP backbone, and (IV) DNP backbone encircled by $\left(\mathrm{CBPQT}^{4+}\right)\left(\mathrm{PF}_{6}{ }^{-}\right)_{4}$ that are thiol bridged to $\mathrm{Au}(111)$ electrodes with $195 \AA^{2}$ unit cell area and $19 \AA$ gap size (see EPAPS figures [12]). We used the same density-functional and matrix Green's calculational parameters as for the full [2]catenane case. Note that the relatively large cell size should reduce the intermolecular interactions or the monolayer packing effect.

The PDOS and transmission data of segment models are shown in Figs. 3(a)-3(d). Important features are that (i) TTF HOMO is higher and closer to $E_{\mathrm{F}}$ than DNP HOMO, and (ii) $\mathrm{CBPQT}^{4+}$ encircling, providing two LUMO levels, pulls down the HOMO level in both cases. These results agree with our previous findings within free molecules [5]. The downward shift of the HOMO transmission with $\mathrm{CBPQT}^{4+}$ encircling results from the positive electrostatic potential exerted by $\mathrm{CBPQT}^{4+}$. Comparing the PDOS of (I + IV) and (III + II) compositions with the full CBPQT@DNP and CBPQT@TTF [2]catenane, respectively, we indeed observe differences resulting from the backbone coupling (through polyethers) and monolayer packing. Noticeable hybridizations exist in the full [2]catenane cases [e.g., significant DNP contribution in the TTF-originated HOMO PDOS peak within CBPQT@TTF [2]catenane of Fig. 2(c)]. Also, the HOMO has the TTF origin for the full CBPQT@TTF [2]catenane while the simple addition of segments (III + II) results in the DNPoriginated HOMO. 

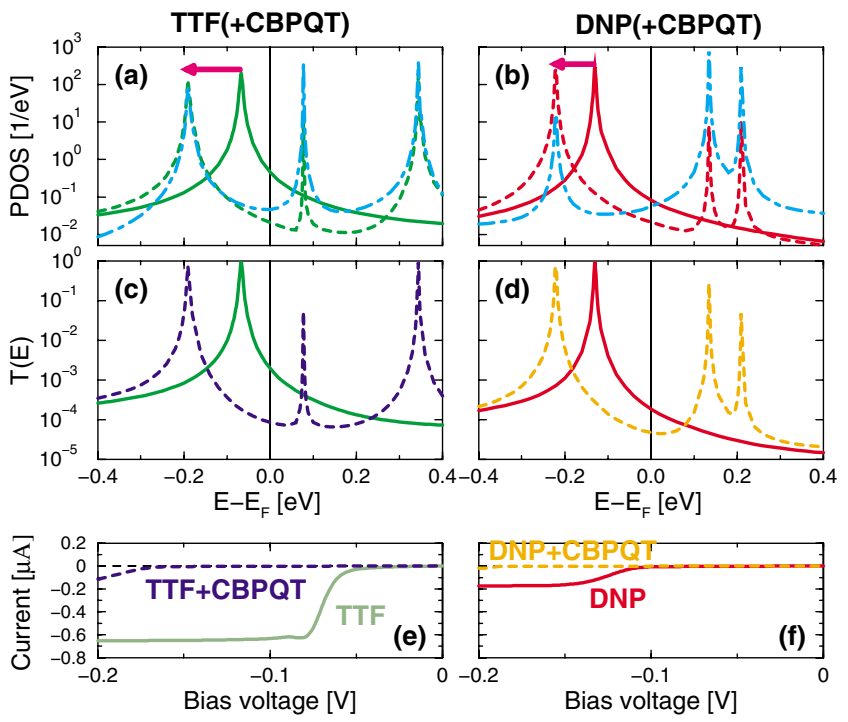

FIG. 3 (color online). (a) TTF PDOS of the segment model I (solid lines), and TTF (dashed lines) and CBPQT ${ }^{4+}$ (dot-dashed lines) PDOS of the segment model II. The arrow represents the TTF peak shift with CBPQT ${ }^{4+}$ encircling. (b) DNP PDOS of the segment model III (solid lines) and DNP (dashed lines) and $\mathrm{CBPQT}^{4+}$ (dot-dashed lines) PDOS of the segment model IV. The arrow represents the DNP peak shift with $\mathrm{CBPQT}^{4+}$ encircling. Transmissions of (c) I (solid lines) and II (dashed lines), and (d) III (solid lines) and IV (dashed lines), and corresponding $I-V$ curves (e) and (f).

Irrespective of such differences, the assignment of the ON/OFF order is consistent between the full and segment models, since the identified switching mechanism involves only the energetic position of the HOMO: Collecting the $I-V$ characteristics of the segments [Figs. 3(e) and 3(f)], we can assign $(\mathrm{I}+\mathrm{IV})(\approx \mathrm{CBPQT} @ \mathrm{DNP}[2]$ catenane $)$ as the ON state and (III + II) ( $\approx$ CBPQT@TTF [2]catenane) as the OFF state. This is valid when the read-bias probes the tunneling status between $E_{\mathrm{F}}-0.07 \mathrm{eV}$ and $E_{\mathrm{F}}-$ $0.22 \mathrm{eV}$ such that the TTF transmission occurs in the former and does not occur in the latter case. So, we conclude that the superposition rule approximately holds. This implies an interesting possibility of designing complicated supramolecular electronic devices based on a theoretical catalog of device elements rather than the expensive experimental feedback procedure [2].

To summarize, we investigated the coherent charge transport characteristics of [2]catenane switches in realistic monolayer configurations. We showed that CBPQT@TTF/DNP is the OFF/ON state of the switch, and the working function of the device can be reduced to a simple picture of the energetic location and movement of several frontier molecular orbitals in which $\mathrm{CBPQT}^{4+}$ acts like an orbital valve. We also demonstrated the utility of the bottom-up approach toward constructing complex supramolecular electronic devices.

We thank Fraser Stoddart, James Heath, Yi Luo, HsianRong Tseng, Amar Flood, and Wei-Qiao Deng for helpful discussions. This work was supported by NSF-NIRT and MARCO-FENA. The facilities of the MSC were supported by ONR-DURIP, ARO-DURIP, NSF-MRI, and the Beckman Institute. Y.-H. Kim also acknowledges the support from IPAM at UCLA.

*Present address: Korea Institute for Advanced Study, 20743 Cheongnyangni 2-dong, Dongdaemun-gu, Seoul, 130722 Korea.

Electronic address: yhkim@kias.re.kr

† Corresponding author.

Electronic address: wag@wag.caltech.edu

[1] C. P. Collier et al., Science 289, 1172 (2000); C. P. Collier et al., J. Am. Chem. Soc. 123, 12632 (2001).

[2] Y. Luo et al., Chem. Phys. Chem. 3, 519 (2002).

[3] V. Balzani et al., J. Org. Chem. 65, 1924 (2000).

[4] R. F. Service, Science 302, 556 (2003); J. R. Heath, J. F. Stoddart, and R. S. Williams, Science 303, 1136 (2004).

[5] Y. H. Jang et al., J. Am. Chem. Soc. 126, 12636 (2004).

[6] W. Deng, R. P. Muller, and W. A. Goddard III, J. Am. Chem. Soc. 126, 13562 (2004).

[7] M. Asakawa et al., Adv. Mater. 12, 1099 (2000).

[8] S. L. Mayo, B. D. Olafson, and W. A. Goddard, J. Phys. Chem. 94, 8897 (1990).

[9] A. K. Rappé and W. A. Goddard, J. Phys. Chem. 95, 3358 (1991).

[10] D. Frenkel and B. Smit, Understanding Molecular Simulation (Academic Press, San Diego, CA, 2001), 2nd ed.

[11] S. S. Jang et al., J. Am. Chem. Soc. 127, 1563 (2005).

[12] See EPAPS Document No. E-PRLTAO-94-045518 for the atomic coordinates of all the models used in this work and the figures of segment models I-IV. A direct link to this document may be found in the online article's HTML reference section. The document may also be reached via the EPAPS homepage (http://www.aip.org/pubservs/ epaps.html) or from ftp.aip.org in the directory /epaps/. See the EPAPS homepage for more information.

[13] P. Schultz, SeqQuest Project (Sandia National Laboratories, Albuquerque, NM, 2003).

[14] J. P. Perdew, K. Burke, and M. Ernzerhof, Phys. Rev. Lett. 77, 3865 (1996).

[15] D. R. Hamann, Phys. Rev. B 40, 2980 (1989).

[16] N. Troullier and J.L. Martins, Phys. Rev. B 43, 1993 (1991).

[17] S. Datta, Electronic Transport in Mesoscopic Systems (Cambridge University Press, Cambridge, 1995).

[18] A. Pecchia and A. D. Carlo, Rep. Prog. Phys. 67, 1497 (2004).

[19] J. Heath et al. This behavior has been confirmed through private communications.

[20] G. Onida, L. Reining, and A. Rubio, Rev. Mod. Phys. 74, 601 (2002). 